\title{
Prevalencia de hepatitis $B$ en un servicio de ginecobstetricia, 1992-1993
}

\author{
Martha Velandia ${ }^{1}$, Fernando de la Hoz ${ }^{1}$, Mancel Martínez ${ }^{1}$, Magdalena Figueroa ${ }^{2}$, \\ Martha Gómez ${ }^{2}$, Sonia Marrugo ${ }^{3}$
}

\section{Resumen}

Se llevó a cabo un estudio de seroprevalencia de la infección por el virus de la hepatitis B (VHB) en el hospital de Honda (Tolima). La población objetivo estaba compuesta por mujeres que consultaron a los servicios de ginecobstetricia de diciembre de 1992 a enero de 1993. A todas se les practicaron pruebas para detectar infección por el VHB (antígeno de superficie, anticuerpo contra el antígeno central y anticuerpo contra el antígeno de superficie). En total, se encuestaron 246 mujeres entre los 15 y los 50 años. La prevalencia encontrada fue de $13 \%$ (32 positivas), la mitad de las personas infectadas (16 pacientes) tenía el patrón de inmunidad adquirida por la infección mientras que el resto mostraba evidencia serológica de infección reciente (14 pacientes portaban el antígeno de superficie o el anticore).

Los factores de riesgo diferían de acuerdo con el estado de infección analizado. En las personas con infección reciente, los factores de riesgo eran: tener tatuajes (CSP $=14,8$ IC 95\% 0,65-332); tener dos y más compañeros sexuales (CSP=4,3 IC 95\% 0,99-12,9 y $\mathrm{CSP}=8,1 \mathrm{IC} 95 \% 1,1-61,1$, respectivamente) $(\mathrm{p}$ de tendencia=0,01). En las personas con infección antigua, el factor de riesgo asociado fue el antecedente familiar de enfermedad hepática (CSP=3,57 IC 95\% 1,19-10,5). Para los infectados (cualquier marcador positivo), el antecedente familiar de enfermedad hepática permanecía como factor de riesgo significativo (CSP=2,44 IC 95\% 1,03-5,75).

El patrón serológico compatible con infección reciente, en nuestro estudio, se relacionó con la actividad sexual, el efecto de dosis-respuesta hallado, entre mayor número de compañeros e infección, corrobora este hallazgo. Esta relación se diluye cuando se analizan los estadios de infección antigua o cuando se toma a todos como infectados.

El uso de los diferentes estadios de infección por VHB como variables dependientes probó ser una manera más eficiente y más específica, desde el punto de vista biológico, de medir factores de riesgo que el considerar sólo como infección a cualquier marcador positivo.

\section{Summary}

A hepatitis B virus infection (HBV) seroprevalency study was carried out in the hospital at Honda (Tolima). The target population was composed of women who had consulted the

1 Programa de Adiestramiento Avanzado en Epidemiología Aplicada, División de Vigilancia Epidemiológica, Instituto Nacional de Salud, Santa Fe de Bogotá, Colombia

2 Hospital San Juan de Dios, Honda, Tolima.

3 Grupo de Virología, Instituto Nacional de Salud, Santa Fe de Bogotá. 
gyno-obstetric services during the months of December 1992 and January 1993. All women in the study were subjected to HBV detection tests (surface antigen, central antigen antibody and surface antigen antibody). A total of 246 women aged between 15 and 50 years of age were questioned. Prevalence was found to be $13 \%$ ( 32 positive); half of those infected had acquired immunity patterns due to infection while the rest presented serological evidence of recent infection (14 patients were surface antigen or anticore carriers).

Risk factors differed according to infection stage analysed. In people with recent infection risk factors were: tattooes ( $\mathrm{CSP}=14.8 \mathrm{Cl} 95 \% 0.65-3.32)$; having two or more sexual partners $(\mathrm{CSP}=4.3 \mathrm{Cl} 95 \% 0.99-12.9$ and $\mathrm{CSP}=8.1 \mathrm{Cl} 95 \% 1.1-61.1$, respectively) ( $\mathrm{p}$ tendency $=0.01)$. In people with former infection, the associated risk factor was hepatic disease family antecedent (CSP $=3.57 \mathrm{CI} 95 \% 1.19-10.5)$. For infected people (any positive marker) the hepatic disease family antecedent remained as the significant risk factor (CSP=2.44 $\mathrm{Cl}$ 95\% 1.03-5.75).

The serological pattern compatible with recent infection, in this study, was related to sexual activity. The effect of dose-response findings, amongst greater number of partners, corroborated this finding. This relationship is diluted when former infection stages are analy-sed or when all cases are taken as being infected.

The use of different stages of HBV infection as dependent variables proved to be a more efficient and specific means of measuring risk factors, from the biological point of view, than taking only infection as a positive marker.

El virus de la hepatitis B (VHB) es un patógeno capaz de producir tanto infección aguda como crónica, siendo esta última la mayor causa de cirrosis y carcinoma hepatocelular en el mundo (1-3). Este virus está ampliamente distribuido en todo el mundo, pero, su prevalencia y modo de transmisión varían entre áreas geográficas: en países con una baja tasa de infección, la transmisión sexual es la forma de transmisión más informada (4-6); en países donde la infección por el VHB es altamente endémica, la infección se adquiere durante el período perinatal o la niñez temprana portransmisión vertical uhorizontal (7-9).

En Colombia, hay zonas reconocidas de alta, media y baja endemicidad para el VHB (10), pero no se han establecido claramente cuáles son los factores de riesgo de transmisión en cada una de ellas. En zonas de alta endemicidad, como la Amazonia y la Sierra Nevada de Santa Marta, el mecanismo de transmisión que se ha informado ha sido el horizontal durante la infancia: contacto con un familiar ictérico, hacinamiento o muerte de un familiar por hepatitis $(11,12)$, mientras que en las áreas de baja endemicidad, la forma de transmisión es primordialmente sexual; sin embargo, existen en nuestro país pocos estudios que reafirmen esta última aseveración (13).
Durante 1992, se presentó un brote de hepatitis B en la cárcel de Honda, Tolima, y la investigación de campo realizada documentó que la transmisión ocurrió por vía sexual, principalmente. En este estudio, se demostró que la transmisión no se limitó a este centro penitenciario sino que se extendió al resto de la comunidad (13). Por ello, nos propusimos establecer un sistema de vigilancia centinela entre usuarias del servicio de ginecobstetricia del hospital de Honda. El sistema de estudio centinela favorece la recolección de datos de prevalencia de la infección de una enfermedad en una población seleccionada, a bajo costo, con resultados inmediatos y de manera relativamente fácil. Este es el informe del primer año de seguimiento del sistema, el primero en el país que pretende evaluar la magnitud del problema en esta población cautiva y su relación con los factores de riesgo en cada uno de los estadios serológicos de la infección.

\section{Materiales y métodos}

\section{Tipo de estudio y población}

Honda está situada sobre el río Magdalena y pertenece al departamento del Tolima; para 1993, contaba con una población proyectada de 27.237 habi- 
tantes con 8.919 mujeres mayores de 15 años, aproximadamente $(14,15)$. Se realizó un estudio de prevalencia entre todas las mujeres residentes en Honda que consultaron entre diciembre de 1992 y enero de 1993 al servicio de ginecobstetricia del hospital por planificación familiar, atención de parto o control prenatal usando el sistema centinela, ligado, no anónimo. Se prefirió este método a la encuesta con muestreo poblacional dadas las dificultades logísticas existentes.

\section{Pruebas de laboratorio y encuesta de riesgo}

A cada mujer que consultó y aceptó participar en el estudio, bajo consentimiento firmado, se le extrajo una muestra única de $10 \mathrm{~mL}$ de sangre que fue enviada al Laboratorio de Virología del Instituto Nacional de Salud donde se practicaron pruebas por la técnica de ELISA para antígeno de superficie ( $\mathrm{HBsAg})$, anticuerpo contra el antígeno de superficie (antiHBs) y anticuerpo contra el antígeno central (antiHBc o anticore). Al mismo tiempo que se tomaba la muestra, un investigador entrevistaba a cada persona individualmente, asegurándosele el anonimato. Se aplicaba una encuesta estructurada en la que se indagaba por los siguientes antecedentes: edad, enfermedades supurativas, transfusiones, cirugías, tratamientos odontológicos, tatuajes, número de compañeros sexuales y antecedentes familiares de hepatitis, cáncer hepático o cirrosis.

\section{Definiciones}

Las pacientes fueron clasificadas según su estado serológico como susceptibles, si tenían los tres marcadores negativos o infectados si tenían, por lo menos, un marcador positivo ( $\mathrm{HBsAg}$, antiHBc, antiHBs). Este, a su vez, se consideraba como infección reciente si estaba presente el HBsAg o el antiHBc; infección antigua si estaba presente el antiHBs y el antiHBc que, además, se consideró como inmunidad por infección y como inmunidad adquirida por vacuna si tenía presente el antiHBs solamente. Las personas con este último patrón serológico fueron excluidas del análisis. Cada una de estas categorías se comparó por aparte contra el grupo de susceptibles, respecto de las variables recogidas en la encuesta.

\section{Análisis estadístico}

Se realizó un análisis univariado para determinar la frecuencia de cada una de las características estudiadas. Luego, se midió la asociación de éstas con la infección comparando su frecuencia entre las personas con diferentes estadios de infección, utilizando para ello tablas de $2 \times 2$, obteniendo razones de prevalencia (RP) y los intervalos de confianza del $95 \%$ (IC 95\%). La relación dosis respuesta fue evaluada usando el $\mathrm{X}^{2}$ de tendencia $y$, en la regresión logística, tratando las variables categóricas como ordinales.

Se usó el análisis de regresión logística para estimar las asociaciones ajustando por todas las variables (edad, ocupación, antecedentes personales de enfermedades supurativas, transfusiones, cirugías, tratamientos odontológicos, tatuajes, número de compañeros sexuales y antecedentes familiares de enfermedad hepática). Las variables categóricas (edad y compañeros sexuales) se convirtieron en $\mathrm{K}-1$ variables indicadoras y juntas fueron incluidas en cada modelo. Este modelo comparó las prevalencias de categorías de las variables independientes, usando los cocientes de suertes de prevalencia (CSP) con cada uno de los estadios de infección y se calcularon sus IC del $95 \%$. Estos CSP estiman cercanamente las razones de prevalencia.

\section{Resultados}

Se encuestaron serológicamente 246 mujeres entre 15 y 50 años, con una media de 25 años. El $50 \%$ (125) de las encuestadas fueron reclutadas para el estudio cuando consultaron para atención de parto, $48 \%$ durante el control prenatal y $2 \%$ en la consulta de planificación familiar. En cuanto a los factores de riesgo evaluados en la encuesta, $83 \%$ (206) se dedicaba al hogar, $27 \%$ (67) tenía el antecedente familiar de enfermedad hepática, 19\% (48) se había practicado algún tratamiento odontológico en los últimos 6 meses, 13\% (31) había sido intervenida quirúrgicamente y $3 \%$ (8) se había practicado tatuajes. El 69\% (170) había tenido un solo compañero sexual durante toda la vida, $21 \%(51)$ dos, $5 \%$ (12) tres y $1 \%$ (2) cuatro, que fue el número máximo de compañeros sexuales informados en las entrevistas.

En los resultados serológicos, se encontró que 214 mujeres ( $87 \%$ ) eran seronegativas para cualquier marcador de infección, mientras que 32 (13\%) eran positivas, al menos, para uno de ellos: 
$10(4,1 \%)$ eran positivas para el antígeno de superficie; $4(1,6 \%)$ únicamente para el anticore; 16 $(6,5 \%)$ tenían inmunidad adquirida por la infección. Dos personas tenían positivo sólo el anticuerpo contra el antígeno de superficie, lo cual es compatible con un estado de vacunación contra VHB y fueron excluidas del análisis de factores de riesgo.

Los cuadros muestran las frecuencias de infección de acuerdo con los marcadores empleados para medir infección por VHB, según los distintos factores de riesgo. Se observa que la fuerza de la asociación con los factores de riesgo estudiados difiere de acuerdo con el estado de infección analizado, como describiremos en las siguientes secciones.

Infección reciente: $\mathrm{HBsAg}(+)$ o anticore $(+)$ : en el cuadro 1 se observa que la prevalencia de infección reciente entre las mujeres que han tenido más de un compañero sexual durante toda su vida, es tres veces mayor (10\%) que las que tienen un sólo compañero y 4,5 veces mayor (15\%) entre las que han tenido más de dos compañeros sexuales en su vida. Las razones de prevalencia en el análisis bivariado muestran que el riesgo de infección reciente se incrementa de tres a casi cinco veces por tener más de uno y más de dos compañeros sexuales (RP:3,08 IC 95\% 0,93-10,2 y
$\mathrm{RP}=4,7 \mathrm{IC} 95 \%$ 1,02-22,1), respectivamente. Entonces, la prevalencia de infección reciente pareciera incrementarse linealmente, según el número de parejas sexuales (valor de $\mathrm{p}$ de $\mathrm{X}^{2}$ de tendencia $=0,01$ ). La prevalencia de infección reciente entre los que se practicaron tatuajes es de $28,5 \%$ contra $5,0 \%$ entre los que no se los practicaron; el riesgo de infección se incrementa 5 veces (RP=5,14 IC 95\% 1,4-18,8). En el análisis bivariado, el incremento en la prevalencia de infección y la edad es significativa estadísticamente (valor de $\mathrm{X}^{2}$ de tendencia $=0,04$ ). Al introducir las personas con infección reciente como variable dependiente en el modelo de regresión logística controlando por todas las variables, se sostenían los mismos factores de riesgo: haberse practicado tatuajes: incrementándose el riesgo 14 veces, pero los intervalos de confianza se vuelven inestables (CSP=14,83 IC 95\% 0,65-332); tener más de un compañero sexual cuadriplica el riesgo ( $\mathrm{CSP}=4,33$ IC $95 \%$ 0,99-12,9) y tener más de dos compañeros sexuales eleva el riesgo a 8 veces $(C S P=8,19$ IC $95 \% 1,1-61,1$ ) valor de $p$ de tendencia $=0,01 ; \sin$ embargo, la edad como factor de riesgo desaparece al controlar por las demás variables estudiadas ( $p$ de tendencia $=0,98$ ).

Infección antigua: antiHBc (+) y antiHBs (+): en el cuadro 2 se observa que la prevalencia de in-

Cuadro 1. Asociación entre los factores de riesgo estudiados e Infección reciente, HBsAg (+) y antiHbc (+), Honda, 1992-1993.

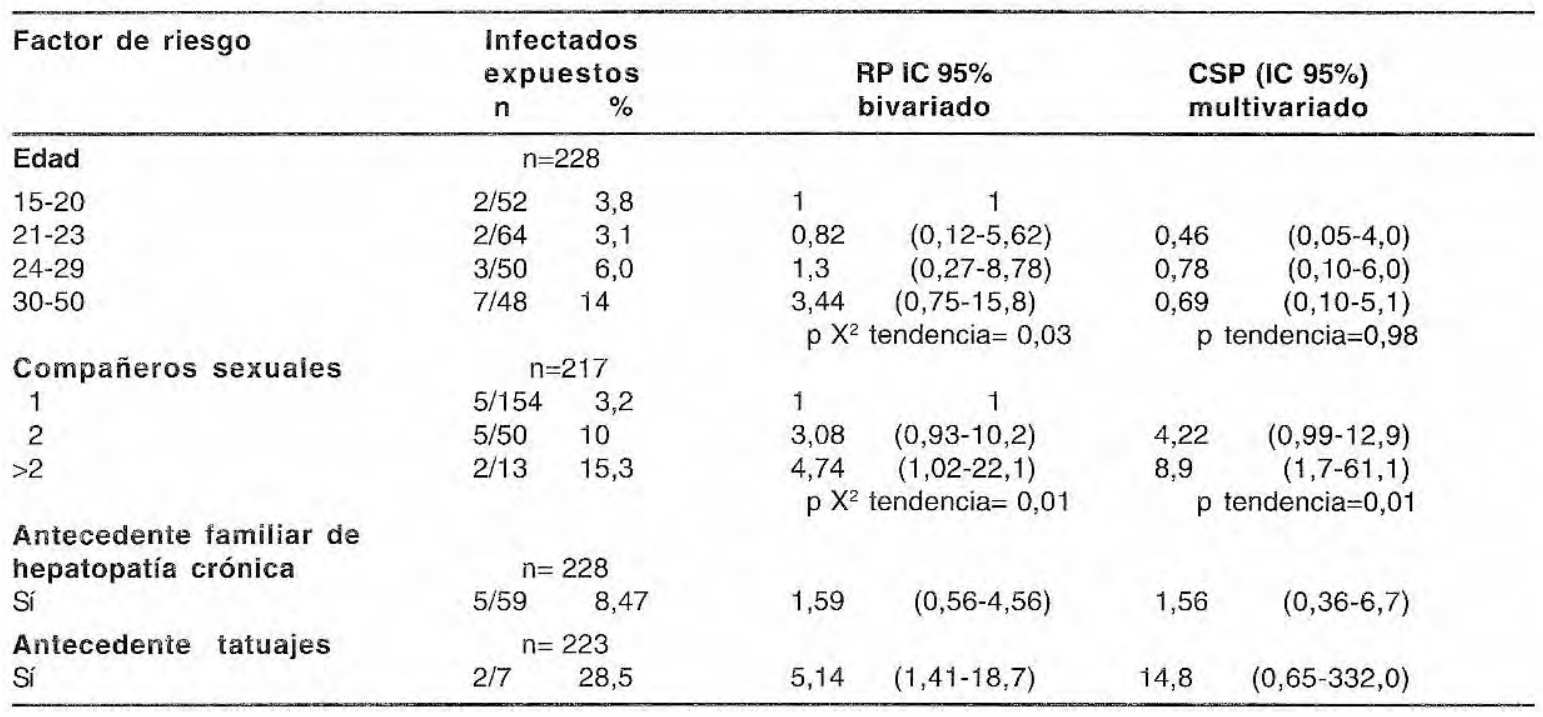


fección antigua es tres veces mayor entre las pacientes que tenían el antecedente familiar de ictericia o enfermedad hepática crónica positivo que entre aquéllas sin este antecedente (12,9\% vs. $4,7 \%$ ) lo que incrementa el riesgo casi tres veces $(R P=2,71$ IC 95\% 1,06-6,91). Usando esta variable dependiente, no existe asociación estadísticamente significativa entre el número de parejas sexuales y la infección. De igual manera, el antecedente de haberse practicado tatuajes pierde significancia estadística. En la regresión logística, tomando como variable dependiente a la infección antigua y controlando por las otras variables, se encuentra que el antecedente familiar de ictericia o enfermedad hepática crónica se mantiene como factor de riesgo (CSP $=3,37$ IC 95\% 1., 10-10) y desaparece el número de compañeros sexuales y los tatuajes.

Infectados: por lo menos, un marcador positivo: cuando la definición de caso incluye las dos definiciones de caso anteriormente descritas, el haberse tatuado ( $\mathrm{RP}=3,21 \mathrm{IC} 95 \% 1,23-8,4)$ y el antecedente familiar de ictericia o enfermedad hepática crónica ( $\mathrm{RP}=2,02 \mathrm{IC} 95 \% 1,04-3,93)$, permanecían como factores de riesgo significativos en el análisis bivariado, como se observa en el cuadro 3. Cuando se tomaba a los infectados como variable dependiente en la regresión logística, el haberse tatuado perdía significancia estadística (CSP=2,88 IC 95\% 0,39-20,9) y el antecedente familiar de enfermedad hepática crónica permanecía como el único factor de riesgo estadísticamente significativo (CSP=2,44 IC 95\% 1,03-5,75). Usando esta definición de caso, también se diluye la relación dosis-respuesta entre compañeros sexuales e infección, observada cuando analizamos el riesgo de infección reciente.

\section{Discusión}

La prevalencia encontrada en esta población es baja según los valores considerados por la Organización Mundial de la Salud (1). Sin embargo, se debe tener en cuenta que el estudio fue realizado en una población hospitalaria y que la prevalencia de infección por VHB hallada con este sistema es aplicable únicamente a las usuarias del hospital; la elección de las mujeres consultantes al servicio de ginecobstetricia como población centinela fue hecha con base en la sospecha que se tenía del incremento en la transmisión del VHB en la población; si la prevalencia estaba incrementando, muy probablemente en uno de los grupos en los que se observaría sería en las mujeres, además de ser este un grupo cautivo de muy fácil acceso.

En el Estudio Nacional de Salud, 1977-1980, realizado por el INS, se encontró, para la región cen-

Cuadro 2. Asociación entre los factores de riesgo estudiados e infección antigua, antiHBc (+) y antiHBs (+), Honda, 199293.

\begin{tabular}{|c|c|c|c|c|c|c|}
\hline \multirow[b]{2}{*}{ Factor de riesgo } & \multicolumn{2}{|c|}{$\begin{array}{l}\text { Infectados } \\
\text { expuestos }\end{array}$} & \multirow{2}{*}{\multicolumn{2}{|c|}{$\begin{array}{l}\mathrm{RP} I C 95 \% \\
\text { bivariado }\end{array}$}} & \multirow{2}{*}{\multicolumn{2}{|c|}{$\begin{array}{l}\text { CSP (IC 95\%) } \\
\text { multivariado }\end{array}$}} \\
\hline & $\mathrm{n}$ & $\%$ & & & & \\
\hline Edad & \multicolumn{2}{|c|}{$\mathrm{n}=230$} & & & & \\
\hline $15-20$ & $8 / 60$ & 15,3 & 1 & 1 & & \\
\hline $21-23$ & $2 / 66$ & 3,1 & 0,23 & $(0,05-1,03)$ & 0,21 & $(0,04-1,1)$ \\
\hline $24-29$ & $3 / 53$ & 6,0 & 0,42 & $(0,12-1,52)$ & 0,38 & $(0,08-1,7)$ \\
\hline $30-50$ & $3 / 51$ & 6,2 & \multicolumn{2}{|c|}{$\mathrm{p} \mathrm{X}^{2}$ tendencia $=0,18$} & \multicolumn{2}{|c|}{$p$ tendencia $=0,37$} \\
\hline Compañeros sexuales & \multicolumn{2}{|c|}{$n=221$} & & & & \\
\hline 1 & $15 / 164$ & 9,4 & 1 & 1 & & \\
\hline 2 & $1 / 46$ & 2,17 & 0,24 & $(0,03-1,75)$ & 0,17 & $(0,02-1,54)$ \\
\hline A to & $0 / 11$ & 0 & \multicolumn{2}{|c|}{$\begin{array}{l}\text { no calculable } \\
p X^{2} \text { tendencia }=0,06\end{array}$} & \multicolumn{2}{|c|}{$\begin{array}{l}\text { no calculable } \\
p \text { tendencia }=0,09\end{array}$} \\
\hline $\begin{array}{l}\text { Antecedente familiar de hepatopatía } \\
\text { crónica }\end{array}$ & \multicolumn{2}{|c|}{$n=230$} & & & & \\
\hline $\mathrm{Si}$ & $8 / 62$ & 12,9 & 2,71 & $(1,06-6,91)$ & 3,37 & $(1,10-10)$ \\
\hline $\begin{array}{l}\text { Antecedente de } \\
\text { tatuajes }\end{array}$ & \multicolumn{2}{|c|}{$n=225$} & & & & \\
\hline Sí & $1 / 6$ & 16,6 & 2,43 & $(0,38-15,5)$ & 2,90 & $(0,12-70,1)$ \\
\hline
\end{tabular}


Cuadro 3. Asociación entre los factores de riesgo estudiados e infectados, HBsAg $(+)$ o anticore $(+)$ o antiHBs $(+)$, Honda, 1992-1993.

\begin{tabular}{|c|c|c|c|c|c|c|}
\hline \multirow[b]{2}{*}{ Factor de riesgo } & \multicolumn{2}{|c|}{$\begin{array}{l}\text { Infectados } \\
\text { expuestos }\end{array}$} & \multicolumn{2}{|c|}{$\begin{array}{r}\text { RP IC } 95 \% \\
\text { bivariado }\end{array}$} & \multicolumn{2}{|c|}{$\begin{array}{l}\text { CSP (IC 95\%) } \\
\text { multivariado }\end{array}$} \\
\hline & n & $\%$ & & & & \\
\hline Edad & \multicolumn{2}{|c|}{$\mathrm{n}=244$} & & & & \\
\hline $15-20$ & $10 / 62$ & $(16,1)$ & 1 & 1 & & \\
\hline $21-23$ & $4 / 68$ & $(5,80)$ & 0,36 & $(0,12-1,10)$ & 0,28 & $(0,08-1,0)$ \\
\hline $24-29$ & $6 / 56$ & $(10,7)$ & 0,66 & $(0,26-1,71)$ & 0,56 & $(0,2-1,78)$ \\
\hline \multirow[t]{2}{*}{$30-50$} & $10 / 58$ & $(17,2)$ & 1,07 & $(0,48-2,38)$ & 0,51 & $(0,2-1,76)$ \\
\hline & & & \multicolumn{2}{|c|}{$p X^{2}$ tendencia $=0,66$} & \multicolumn{2}{|c|}{$p$ tendencia $=0,43$} \\
\hline Compañeros sexuales & \multicolumn{2}{|c|}{$n=233$} & & & & \\
\hline 1 & $20 / 169$ & $(11,8)$ & 1 & 1 & & \\
\hline 2 & $6 / 51$ & $(11,7)$ & 0,99 & $(0,42-2,34)$ & 1,17 & $(0,40-3,3)$ \\
\hline \multirow[t]{2}{*}{$>2$} & $2 / 13$ & $(15,3)$ & 1,30 & $(0,34-4,96)$ & 1,29 & $(0,22-7,17)$ \\
\hline & & & \multicolumn{2}{|c|}{$p X^{2}$ tendencia $=0,79$} & \multicolumn{2}{|c|}{$p$ tendencia $=0,68$} \\
\hline \multirow{2}{*}{$\begin{array}{l}\text { Antecedente familiar de } \\
\text { hepatopatía crónica } \\
\text { Sí }\end{array}$} & \multicolumn{2}{|c|}{$n=244$} & & & & \\
\hline & $13 / 67$ & $(19,4)$ & 2,02 & $(1,04-3,93)$ & 2,44 & $(1,03-5,7)$ \\
\hline \multirow{2}{*}{$\begin{array}{l}\text { Antecedente de tatuajes } \\
\text { Sí }\end{array}$} & \multicolumn{2}{|c|}{$n=239$} & & & & \\
\hline & $3 / 8$ & $(37,5)$ & 3,21 & $(1,23-8,40)$ & 2,88 & $(0,39-20,9)$ \\
\hline
\end{tabular}

tral constituida por los departamentos de Tolima, Huila, Caldas y Antioquia, una prevalencia promedio de antígeno de superficie de $7,1 \%$, la cual es bastante más alta que el $4 \%$ encontrado en nuestro estudio (9). La razón de las diferencias puede estar en el muestreo, dado que el primer estudio fue realizado sobre una muestra representativa de la población de cuatro departamentos y puede que la precisión para inferir sobre una sola población sea poca.

Cuatro por ciento de las mujeres encuestadas tenía el antígeno de superficie positivo, lo cual predice un alto riesgo de infección para los recién nacidos de estas mujeres, con las complicaciones a largo plazo de cáncer hepatocelular y cirrosis (1). Este patrón serológico es compatible con el estado de infección reciente relacionada posiblemente con la actividad sexual, el efecto de dosisrespuesta entre mayor número de compañeros e infección hallado en nuestro estudio ( $p$ de tendencia método multivariado $=0,01$ ) corrobora este hallazgo. Es de resaltar la asociación de infección con los tatuajes, los cuales a pesar de ser un mecanismo plausible de infección no es evaluado a menudo en los estudios publicados en la literatura universal $(16,17)$. Esta es una práctica muy frecuente en ciertos estratos socioeconómicos de nuestro medio y, por tanto, puede ser un vehículo importante de transmisión del VHB, alternativamente.
La medición válida de factores de riesgo en hepatitis B es un ejercicio arduo por las características serológicas de la infección, dado que existen en la historia natural de la enfermedad tres estadios serológicos bien identificados, es posible que los factores de riesgo difieran para cada uno de ellos; a pesar de esto, en la literatura generalmente no se hace esta distinción sino que se comparan estos factores contra el hecho de ser positivo para, al menos, uno de los tres $(17,19)$, o se toma en consideración exclusivamente la presencia de HBsAg $(16,18)$.

A pesar del posible sesgo de mala clasificación que pudo haber sido introducido en el estudio por no usar métodos de laboratorio que facilitaran la discriminación entre la infección antigua y la reciente (por ejemplo, lgM antiHBc), el uso de los diferentes estadios de infección por VHB como variables dependientes probó ser una manera más eficiente y más específica, desde el punto de vista biológico, de medir factores de riesgo que el considerar solo como infección a cualquier marcador positivo. La homogeneidad de la condición bajo estudio es, según una corriente del pensamiento epidemiológico, una ventaja para establecer cuantitativamente relaciones causales y evaluar el riesgo. En este caso, es evidente que, al mezclar infecciones recientes con antiguas, es más difícil establecer las conexiones entre relaciones sexuales e infección por dos razones: 1) 